\title{
On Cooperatively Creating Dynamic Ontologies
}

\author{
Eva Gahleitner \\ FAW Software Engineering gGmbH \\ Hauptstrasse 119 \\ A-4232 Hagenberg \\ +4372363343771 \\ egahleitner@faw.uni-linz.ac.at \\ Jürgen Palkoska \\ Institute for Applied Knowledge Processing \\ Altenbergerstr. 69 \\ A-4040 Linz \\ +4372363343770 \\ jpalkoska@faw.uni-linz.ac.at
}

\author{
Wernher Behrendt \\ Salzburg Research Forschungsgesellschaft mbH \\ Jakob Haringer Str.5 / III \\ A-5020 Salzburg \\ +43662 2288200 \\ wernher.behrendt@salzburgresearch.at
}

\author{
Edgar Weippl \\ Institute for Software Technology and Interactive \\ Systems Information \& Software Engineering Group \\ Favoritenstrasse 9-11/188 \\ A-1040 Vienna \\ +43158801 18888 \\ weippl@ifs.tuwien.ac.at
}

\begin{abstract}
Collaborative construction of ontologies is still hampered by immature methodologies and by tools which are insufficient for domain experts who are not at the same time, knowledge engineers. The DynamOnt project has set out to develop a methodology for collaboratively creating group ontologies which evolve over time as well as in space and internal complexity. The project also seeks to identify requirements and specifications for tools which will support the construction of such dynamically evolving ontologies. Major issues are the guidance of knowledge workers towards soundly constructed ontologies with the help of upper level ontologies and an exploration into language issues - in our case the scenario for constructing ontologies, if German is the language of choice for the domain experts. We also investigate the relationship between terminology and ontology, which we view as a bridge between linguistically motivated and IT motivated standardisation of conceptual models. We envisage knowledge workers' environments of the future to be tightly integrated systems with their hypertextual capabilities being controlled by ontologically sound interaction and navigation models.
\end{abstract}

Categories and Subject Descriptors: H.4.m [Information Systems]: Miscellaneous; H.3.5 [Information Storage and Retrieval]: Online Information Services - Web-based Services

General Terms: Algorithms, Design, Standardization, Theory

Keywords: DynamOnt, Cooperative and Collaborative

Environments, Dynamic Ontologies, Upper Level Ontologies, Terminologies

DynamOnt project is supported by the Austrian Federal Ministry for Transport, Innovation, and Technology under the FIT-IT contract FFG $809256 / 5512$ and factline Webservices and ProCom Strasser who are industrial application partners in the project.

Permission to make digital or hard copies of all or part of this work for personal or classroom use is granted without fee provided that copies are not made or distributed for profit or commercial advantage and that copies bear this notice and the full citation on the first page. To copy otherwise, or republish, to post on servers or to redistribute to lists, requires prior specific permission and/or a fee.

$H T^{\prime} 05$, September 6-9, 2005, Salzburg, Austria.

Copyright 2005 ACM 1-59593-168-6/05/0009...\$5.00.

\section{INTRODUCTION}

Hypertext has often been considered as a means for sharing knowledge among a community of users. Currently, project teams use Web-based platforms to organize their work. Definitions, facts and documents are linked to each other in order to add relevant contextual information to the text itself.

However, domain experts from non-IT areas of expertise require considerable time to develop a common understanding during an ad-hoc process. The construction and use of ontologies leads to tighter definitions of agreed semantics and has therefore become the favoured approach towards improving human interaction with computers and human-to-human interaction via computers. Nevertheless, ontology building is still much more a craft rather than an engineering discipline. Each development team usually follows its own set of principles, design criteria, and phases.

Furthermore collaboration of distributed knowledge communities is a rapidly increasing application field (e.g. international enterprises, scientific research teams, e-learning communities). For efficient collaboration the mutual understanding of information is essential. A systematic approach to gain this common understanding is the dynamic creation of ontologies, leading to a more efficient use of shared information resources. Currently, the creation of high-quality ontologies is a very time consuming and expensive task. Therefore, such ontologies are available only for a few thematic fields, where there is hope for significant economies of scale (e.g. health sector, tourism, insurance sector). Although several ontology development methodologies have been introduced in the past, there are still methodologies missing that enable knowledge workers (who are not ontology building experts) to dynamically create sound ontologies in collaborative work environments. In this context "dynamic" means that these ontologies can be extended and refined over time, possibly by other non-IT experts. They can evolve to become more axiomatised, and can be personalised and localised by individuals or groups without losing touch with the community's preferred interpretation.

The DynamOnt project aims at generating methodologies and toolsets that can be applied to fulfil the above requirements. Users will be able to do fast prototyping of ontologies while adhering to principles of sound ontology engineering through the use of con- 
sistency checks and guided questions provided by the methodology. The abbreviation DynamOnt stands for Dynamic Ontologies.

The remainder of the paper is organized as follows: section 2 gives an overview of related work on methodologies for ontology development and describes the need for collaboration in distributed knowledge domains. Section 3 defines the objectives of DynamOnt and its methodology. Finally, section 4 concludes the paper and gives an outlook on further research activities within the project.

\section{STATE OF THE ART OF ONTOLOGY DEVELOPMENT}

Currently, analyzing application domains and afterwards building valid and useful ontologies for those domains is a labour-intensive task for specialists (ontology engineers). For gaining and modelling knowledge in ontologies several methodologies have been introduced in the last years. OnToKnowledge [1] is based on software engineering lifecycle models starting from a requirement analysis and reaching to organising the maintenance of the resulting ontology. The Skeletal Methodology [2] comprises a set of simple guidelines for developing ontologies. Methontology [3] offers detailed support in development-oriented activities, and describes project management activities. Other methodologies are more suitable for reviewing existing ontology structures. For instance OntoClean [4] tests the ontological adequacy of taxonomic links in ontologies (e.g. if the subsumption relations are correct).

All methodologies have in common that the ontology designers must have well consolidated knowledge in the area of ontology engineering. Furthermore, most approaches assume that, when designing ontologies, there will be enough knowledge available about the applied concepts and relations already at the beginning of the modelling process. The modelling itself is done by ontology designers in cooperation with domain knowledge workers. This separate process of creating ontologies may lead to misunderstandings and inconsistencies if the ontology designer is not fully involved into the domain work.

\subsection{The need of collaboration}

Ontologies help to share knowledge within an organization or in distributed professional communities and to reach common goals. Taking the challenge of creating ontologies away from dedicated ontology designers and making it a cooperative modelling task done by the community members will lead to a novel ontology engineering process.

That process has to address the various forms of knowledge models within a community and their projects. Depending on the progress of the projects there exist different conceptualizations at different levels of formality, starting with the simplest one [5]: glossaries (textual descriptions of terms), taxonomies (hierarchically structured terms), thesauri (interrelations of terms), DB or OO schemas (formal computational models), and ontologies (axiomatised theories). In addition to the more or less explicit and formal conceptualization of a domain by ontologies (or various conceptualization steps towards them), their designations in a certain cooperative domain and its special language (terminology) must be considered. Terminologies are similar to ontologies but their main purpose is to be read by humans rather by computer applications. They focus more on their linguistic and communicative functions and are not (yet) fully ontologised [6]. The advantage of terminologies is that they provide far more nuances of differing semantics between similar concepts in different (domain) languages than computer scientists building ontologies. Therefore, terminologies like WordNet [11] can help to understand the subtle differences between generic concepts and language-dependent ones; they will be able to tackle new languages and their associated cultures and markets.

Creating ontologies in such a cooperatively and dynamic way requires an ontology methodology to allow graceful migration from weakly structured glossaries to highly structured axiomatic theories, and transforming linguistic expressions into formal ontological statements.

\subsection{Levels of ontologies}

Ontologies are the fundamental infrastructure for modern interoperable information systems and are the foundation for implementing the so-called Semantic Web. Different levels of conceptualization generality lead to different kinds of ontologies. A common distinction is made between Upper Level Ontologies (ULO) and Domain Ontologies.

Domain ontologies describe the knowledge of a certain domain (e.g. for tourism, medicine, etc.). Upper level ontologies describe domain-independent concepts. They comprise such highly general categories as: time, space, inheritance, instantiation, identity, processes, events, attributes etc. ULOs determine a framework for the construction of domain-specific and generic ontologies and are concerned with much-debated philosophical problems [7].

Some authors define ULO in the context of ontology management systems [1]. According to these systems they state that it could be useful if (lower level) ontologies are grounded in an existing upper level ontology. The upper level ontology captures and models the basic concepts and knowledge that could be re-used in creating new ontologies and in organizing ontology libraries. Representative examples for ULOs are DOLCE [8], SUMO [9], and Sowa's ontology [10].

\section{THE DYNAMONT PROJECT}

The DynamOnt project aims at bringing the ability to develop and maintain sound ontologies to professionals who are not trained knowledge engineers.

\subsection{Objectives of the Research-Project}

We are working towards a solution to the mismatch between three categories of existing tools and methodologies: (1) linguistic tools for terminology handling, (2) ontological modelling tools, and (3) tools for data- or software engineering (e.g. ER, UML, etc). Therefore, our main objectives are

- To develop a methodology that allows knowledge workers who are not IT-specialists and who are also not formal ontologists, to create ad-hoc categorisations or segmentations of arbitrary "knowledge spaces" very fast, without having to compromise on the quality of the evolving conceptual model.

- To converge different traditions within ontology engineering (foundational approaches, linguistic approaches, computer science, IEEE/W3C standards) as well as approaches from terminology management and computational linguistics.

- To research the interaction between hypertextual knowledge spaces and their ontological categorisation during the cooperative working progress (hypertext is structured through ontologies whereas ontologies building depends on existing heterogeneous and interrelated text universes). 
- Interactive guidance of diverse user groups in diverse domains to create sensible lower ontologies.

- To regard the existence of conceptualizations at different levels of formality.

- To incorporate existing glossaries or taxonomies into evolving group-specific ontologies

- To permit knowledge workers to express their ideas and conceptualizations in their native language (in a first step the DynamOnt project will regard the German Language)

\subsection{The Role of Upper Level Ontologies}

One important and critical question when starting a new ontology is determining what facts there are in the domain to be modelled and how they can be modelled in a semantically formalized way. In DynamOnt upper level ontologies act as a starting point for defining lower level ontologies that follow a semantically rich and formal description. Their scope is twofold: firstly guiding the non-ontologist through the intended concepts in the domain, and secondly providing an automatic assistance for developing sound lower level ontologies.

These lower level ontologies will be further linked to domain or sector-specific ontologies (e.g. within the tourism domain there may already exist a specific ontology for city-tourism).

\subsection{The DynamOnt Methodology}

Figure 1 shows an overview of the DynamOnt components. The central idea is the dynamic generation and maintenance of the knowledge model supporting different levels of model rigour ranging from simple project glossaries to formal domain ontologies.

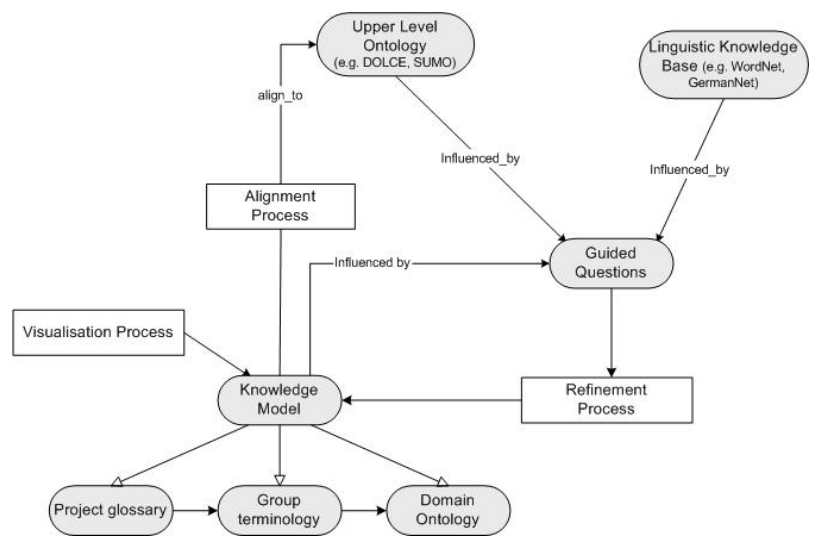

Figure 1 - Schematic overview of DynamOnt

A typical scenario would be a group of people working collaboratively on a project. As part of their work they create knowledge based output, primarily in the form of documents. Since the team consists of experts from different domains, countries and cultures they do not share a common vocabulary, yet. Therefore the users will create a glossary, either starting from existing collections of terminology or by creating new ones. Over time, the glossary increases in size and complexity and additional structural elements will become necessary to manage the evolving term repository.

In DynamOnt, the maintenance of the knowledge model is based on such a refinement process. DynamOnt allows users to organize the entries of the glossary and create a taxonomy. Subsequently additional relations and attributes can be added. Guided questions lead the users to a more structured knowledge model. Using upper level ontologies like DOLCE or SUMO, DynamOnt guides the user by asking questions (e.g. "Is this attribute constant over time?") and automatically detects possible inconsistencies or errors in the ontology.

Furthermore, the refinement process is influenced by linguistic knowledge bases. Therefore, the newly created ontology is related to existing projects such as WordNet[11] or GermaNet[12] by automatically adding links whenever possible and selectively prompting users where required. An adequate visualization will help the user to better understand the given knowledge model.

As a result, the DynamOnt system will lead to tightly coupled document-, content-, terminology- and ontology repositories that will offer large improvements in productivity to heterogeneous, distributed groups of knowledge workers. Moreover, it enables individuals to manage their personal knowledge and content resources.

\section{FUTURE WORK AND CONCLUSION}

We are working on a proof-of-concept prototype for linguistically enabled modelling of domains where the resulting models are aligned with an upper ontology. The prototype will be validated in several real-world scenarios one of which is international collaboration in safety related issues and disaster management.

\section{REFERENCES}

[1] Davies, J., Fensel, and D., Towards The Semantic Web, OntologyDriven Knowledge Management, John Wiley \& Sons Ltd, England, 2002

[2] Uschold, M., and King, M., Towards a Methodology for Building Ontologies, in Proceedings IJCAI-95, Workshop on Basic Ontological Issues in Knowledge Sharing, Canada, 1995

[3] Blázquez, M., Fernández, M., García-Pinar, J.M., Gómez-Pérez, A., Building Ontologies at the Knowledge Level using the Ontology Design Environment, in Proceedings of the Knowledge Acquisition Workshop, KAW98, 1998

[4] Guarino, N., Welty, C., An Overview of OntoClean, in S. Staab, R. Studer (eds.), Handbook on Ontologies, Springer Verlag 2004, pp. 151-159

[5] Guarino, N., 1st Workshop Rappresentazione della conoscenza nel semantic web culturale, W3C Rom, 2004

[6] Budin, G., Ontology-driven Translation Management, EU High Level Scientific Conference Series: Knowledge Systems in Text and Translation, Aarhus, 2003

[7] Degen, W., Herre, H., What is an Upper Level Ontology?, Workshop on Ontologies 2001, Vienna

[8] Gangemi, G. et al, 2002, Sweetening Ontologies with DOLCE, in Proceedings of the 13th International Conference on Knowledge Engineering and Knowledge Management (EKAW'02), Springer, Okt 1-4, Spain, pp. 166-181.

[9] Pease, A., Niles, I., and Li, J. 2002. The Suggested Upper Merged Ontology: A Large Ontology for the Semantic Web and its Applications. In Working Notes of the AAAI-2002 Workshop on Ontologies and the Semantic Web, Edmonton, Canada, July 28August 1, 2002.

[10] Sowa, J., Knowledge Representation, Logical, Philosophical, and Computational Foundations, Brooks/Cole, Pacific Cole, USA, 2000

[11] Miller, G., Beckwith, R., Fellbaum, C., Gross, D., and Miller, K., Introduction to WordNet: An On-line Lexical Database, International Journal of Lexicography, 3(4), 235 -- 244, (1990)

[12] Lemnitzer, L. \& C. Kunze (2002): Adapting GermaNet for the Web, In Proceedings of the First Global Wordnet Conference, Central Institute of Indian Languages, Mysore, India, 21.-25.01.2002, pp. $174-181$ 